\title{
ROMANIAN
}

NEUROSURGERY

\author{
Vol. XXXV | No.1 March 2021
}

\section{Management of a double basilar tip aneurysm}

\author{
A. Chiriac, \\ N. Dobrin, \\ Georgiana Ion, \\ I. Poeata
}




\title{
Management of a double basilar tip aneurysm
}

\author{
A. Chiriac ${ }^{1}$, N. Dobrin ${ }^{2}$, Georgiana Ion ${ }^{2}$, I. Poeata ${ }^{1}$ \\ 1 "Gr. T. Popa" University of Medicine and Pharmacy, Iasi, RomanIA \\ 2 "N. Oblu" Emergency Clinical Hospital, lasi, ROMANIA
}

\begin{abstract}
Complex basilar tip aneurysms are still challenging to secure with coils or stentassisted coiling. Double aneurysms at the basilar bifurcation and the basilar arterysuperior cerebellar artery are a rare particular situation that usually requires more appropriate treatment. This case reports details of our experience with a double basilar tip aneurysm treated in two steps by coiling repair and stent-assisted coiling.
\end{abstract}

\section{INTRODUCTION}

The basilar tip aneurysm (BTA) is still represents a real challenge for a microsurgical approach due to the deep location and proximity to delicate neuro-vascular anatomical structures. Thus, endovascular techniques remain the most commonly used option for the treatment of these lesions.

A double basilar tip aneurysm is a very complex lesion usually characterized by a wide neck that often involves the origins of more vascular branches. This special vascular pathological condition makes even endovascular treatment a major challenge for most interventionists. In such cases, usually preservation techniques using a stent(s) or balloon can be attempted for a one or two steps intervention. Therefore, a proper diagnosis of these double aneurysms and an optimal technique selection are the most important facts in achieving complete obliteration of these lesions. In this paper we intended to report our experience with such rare case of double basilar tip aneurysm successfully treated while reviewing the literature to improve the understanding of this particular clinical situation.

\section{CASE PRESENTATION}

A 38-year-old man was addressed to our emergency room from a service in another hospital on 31 December 2020. A head CT examination at the local hospital suggested Fisher grade 3 subarachnoid haemorrhages and the contrast administration revealed a complex basilar artery aneurysm. The patient was transferred to our hospital for further diagnosis and treatment. At admission patient presented severe headache accompanied by episodes of nausea and

Keywords

double basilar tip aneurysm, stent-assisted coiling

\section{DS}

Corresponding author: A. Chiriac

"N. Oblu" Emergency Clinical Hospital, lasi, Romania

chiriac_a@hotmail.com

Copyright and usage. This is an Open Access article, distributed under the terms of the Creative Commons Attribution Non-Commercial No Derivatives License (https://creativecommons org/licenses/by-nc-nd/4.0/) which permits noncommercial re-use, distribution, and reproduction in any medium, provided the original work is unaltered and is properly cited.

The written permission of the Romanian Society of Neurosurgery must be obtained for commercial re-use or in order to create a derivative work.

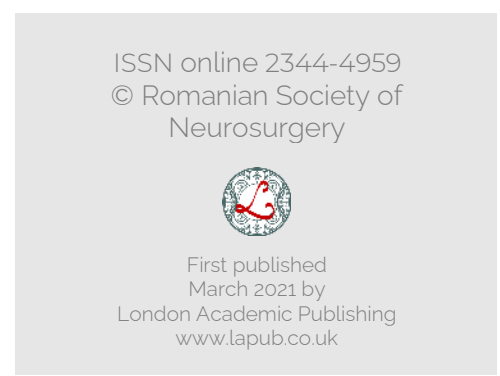


vomiting. The patient had a ten-year history of multiple sclerosis and hypertension with regular treatment. A ruptured large basilar tip aneurysm was confirmed on brain CT angiography, and subsequent subtraction catheter cerebral angiography confirmed a double basilar tip aneurysm at the basilar bifurcation and the basilar artery-superior cerebellar artery junction. A two steps aneurysms embolization treatment was decided. Based on predominant haemorrhage location and aneurysms characteristics the basilar artery-superior cerebellar artery junction aneurysm was considered the ruptured one.

Under general anaesthesia, 6F Merit Medical introducer sheath was placed into the right femoral artery. Using biplane roadmap fluoroscopy a $6 \mathrm{~F}$
Chaperon guiding catheters (Microvention) were advanced over 0.035 glidewire up to the mid-V2 portion of the left vertebral artery. Based on 3D CT images analysis a working angle roadmap for the target aneurysm was obtained. A Prowler 10 microcatheter was then advanced over a 0.014 Transed microwire (Boston Scientific) into the basilar artery-superior cerebellar artery junction aneurysm to allow placement of coils inside the aneurysm dome. Five GALAXY G3 XSFT microcoils (Cerenovus Johnson\&Johnson) were then sequentially inserted and detached into aneurysm. On final angiography, both PCAs were fully saved and the aneurysm was completely packed. The patient was placed into intensive care and received $75 \mathrm{mg}$ clopidogrel and $100 \mathrm{mg}$ aspirin daily.
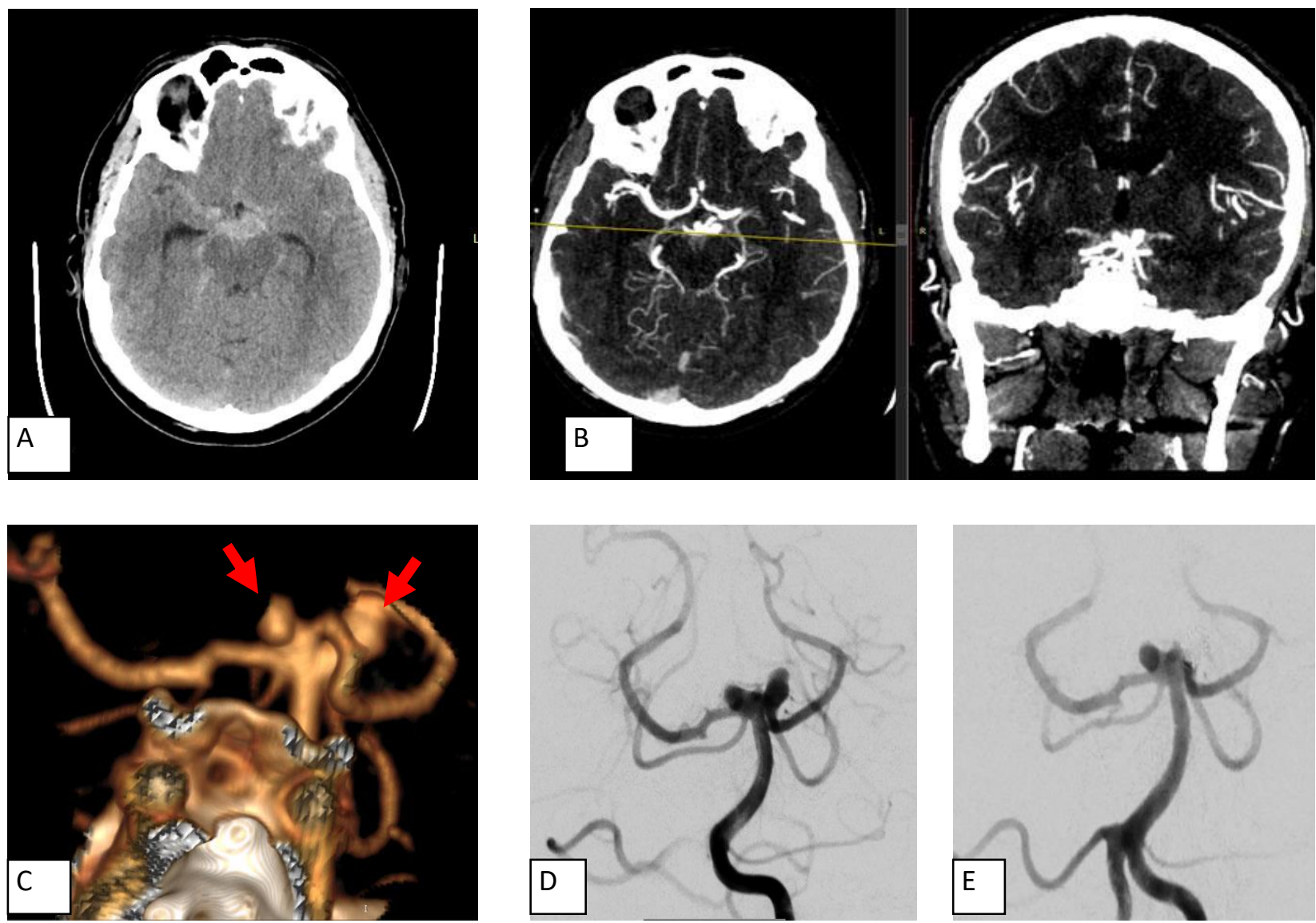

Figure 1. A - First head CT examination at the local hospital showing a SAH; B - brain CT angiography revealing a double basilar tip aneurysm; C - CTA 3D reconstruction; D - DSA in working projection; E - DSA control after basilar artery-superior cerebellar artery junction aneurysm coil occlusion.

After 7 days a second endovascular intervention was decided for associated unrupted basilar bifurcation aneurysm occlusion. A Chaperon guiding catheters
(Microvention) was passed to the right femoral artery and securely advanced up to the half-V2 segment of the left vertebral artery. To pass an Enterprise 2 stent 
(4/23mm), a Prowler Select Plus microcatheter (Codman \&Shurtlett, Inc.) was carefully inserted into the right PCA under the guidance of a 0.014 Transed microwire. The stent was deployed over the Pcom/PCA junction point and both aneurysms necks. The Prowler Select Plus microcatheter is than retracted and a Prowler 10 microcatheter is advanced into the basilar tip aneurysm through the stent using a 0.014 Transed microwire. Three
GALAXY G3 XSFT microcoils were used for complete angiographic occlusion of the aneurysm. The patient was safely returned to intensive care and antihypertensive therapy, antiplatelet therapy, volume expansion therapy, and medication for the prevention of vasospasm were continued. The patient recovered without any complications, and was discarded home 10 days later. He is currently under follow-up as an outpatient.
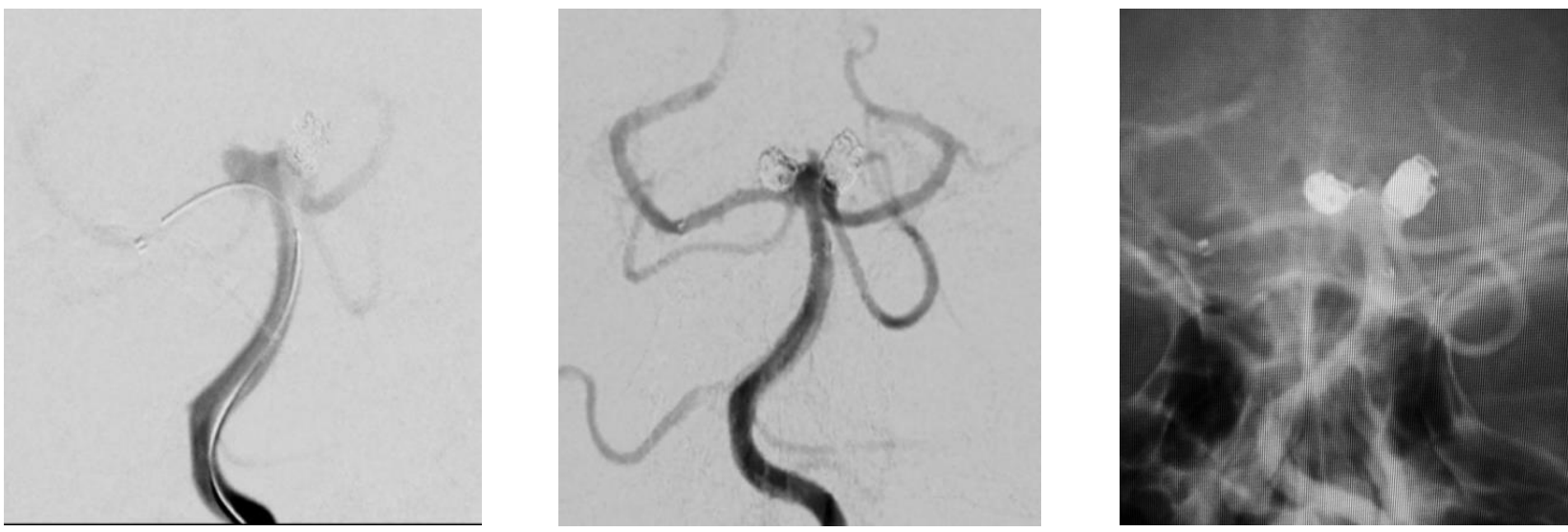

Figure 2. A - Microcatheterization of right PCA for stent placement; B,C - DSA control after stent detachment and trans-stent coil embolization of basilar bifurcation aneurysm.

\section{DISCUSSION}

The basilar tip aneurysm is most commonly associated with a complex anatomical configuration. The main pitfall in the treatment of a basilar tip aneurysm consists in the presence of thalamoperforating arteries and numerous vascular branches in relation with a wide-neck or polylobated aneurysmal dome. Presence of a double basilar tip aneurysm at the basilar bifurcation and the basilar artery-superior cerebellar artery junction is a more complex treatment condition. Tanaka et al reported in 2001 the incidence of double basilar tip aneurysm as $5.3 \%$ and concluded that they were not extremely rare if accurately diagnosed by the use of modern techniques[4]. At the same time, Hernisniemi in an analysis of the article on double basilar aneurysms reported a much lower incidence of them (3 cases of double basilar aneurysms from a total of 112 patients) $[2,3,4]$.

Due to a high reported morbidity of surgical clipping for basilar tip aneurysm, endovascular interventional therapy became commonly used for majority of these clinical situations. For complex basilar tip aneurysm several endovascular techniques such as stent assisted coiling, waffle cone technique and balloon remodelling can be used to increase aneurysm packing during aneurysm coiling [1].

The technique of balloon-assisted coil embolization, firstly introduced by Moret et al., even if it initially showed a high rate of aneurysmal occlusion, many delayed coil compaction and aneurysm recurrence were reported in time.[4,5] The emergence of various stent assisted coiling has offered a safe and effective clinical solution for this type of complex wide-necked bifurcation aneurysm[1,2,3]. These techniques provide scaffolding for stable and dense coil embolization and blood flow diversion effect away from aneurysm. The most representative stent remodelling techniques in coil embolization of basilar apex aneurysms include classical curvilinear stenting technique, double Y-stenting technique, and waffle cone technique. The waffle-cone technique first reported by Horowitz et al has proved in time a satisfactory protection of the parent arteries origins but not an optimal blockage of the aneurysmal neck. Y-stent assisted coiling technique firstly proposed by Chow et al. in 2004, showed a good clinical prognosis for the treatment of basilar tip aneurysm by 
effectively reduction of the aneurysmal neck, protection of the parent arteries and by changing the angle of the blood vessels at the bifurcation. Even if the $Y$ stent configuration has many advantages, it also has several important disadvantages represented by potential thrombus formation and the need for long-term application of double antiplatelet agents. The thrombotic complications associated with this procedure were reported in literature to be approximately $2-21.4 \%[4,6]$.

Given the above, there is clear evidence that the single stenting technique associated to basilar tip aneurysms coil occlusion is much easier and with much lower morbidity rates than the double stenting technique. Representative unique stent remodelling techniques of the basilar apex include a curvilinear or horizontal implant positioning.

Treatment of basilar tip aneurysms by horizontally stenting was firstly described by Cross and colab. They used a unique retrograde approach with horizontally stent deployment to both P1 segments perpendicular to the aneurysm, using the ICA-PCom route[2,3,4]. This approach is completely dependent on the tortuosity and size of the posterior communicating artery. In case of the double basilar apex aneurysms that implicate also the basilar artery-superior cerebellar artery junction, the horizontal stent deployment must start from arterysuperior cerebellar artery to cover the both aneurysms neck.

Our experience as well as the few data reported in the literature has shown that single curvilinear stenting is the most optimal technique in the treatment of double basilar apex aneurysms. This consists of positioning a stent from the P1 segment opposite the junction aneurysm (BA-PCA) over the necks of both aneurysms to the distal segment of the basilar trunk. However, the stent placement strategy is also determined by a precisely differentiation of a double basilar aneurysm from a bilocular aneurysm at the basilar apex pre-intervenionally.

\section{CONCLUSION}

Double wide-neck basilar tip aneurysms are a challenge to repair, but the unique stent assisted coiling technique is proved to be quite effective whether performed in one or two separate sessions.

\section{REFERENCES}

1. Ahmad S. Basilar Tip Aneurysm in Takayasu Arteritis. BJR Case Rep. 2019 Nov 15;5(4):20180114. doi: 10.1259/bjrcr.20180114. PMID: 31938555; PMCID: PMC6945252.

2. Drake CG, Peerless SJ, Hernesniemi JA: Surgery of Vertebrobasilar Aneurysms: London, Ontario, Experience on 1,767 Patients. Vienna, Springer-Verlag, 1995, p 251.

3. Hernesniemi J. Double aneurysms at distal basilar artery: report of nine cases. Neurosurgery. 2001 Jul;49(1):229231. DOI: $10.1097 / 00006123-200107000-00048$.

4. Hongo, K., Tanaka, Y., \& Kobayashi, S. (2001). Double Aneurysms at Distal Basilar Artery: Report of Nine Cases: In Reply. Neurosurgery, 49(1), 230-231.

5. Tanaka, Y., Hongo, K., Nagashima, H., Tada, T., \& Kobayashi, S. (2000). Double aneurysms at distal basilar artery: Report of nine cases. Neurosurgery, 47(3), 587593.

6. Sekhar LN, Tariq F, Morton RP, Ghodke B, Hallam DK, Barber J, Kim LJ. Basilar tip aneurysms: a microsurgical and endovascular contemporary series of 100 patients. Neurosurgery. 2013 Feb;72(2):284-98; discussion 298-9. doi: 10.1227/NEU.0b013e3182797952. PMID: 23147787. 